\title{
Extremely Prolonged Expiration Suppresses Left Amygdala Function; A Pilot Study
}

\author{
Teruhisa Komori* \\ Department of Stress and Health Science, Mie University Graduate School of Medicine, Japan
}

*Corresponding author: Teruhisa Komori, Department of Stress and Health Science, Mie University Graduate School of Medicine, Tsu, Mie 514-8507, Japan.
Received Date: June 01, 2019

Published Date: June 10, 2019

\begin{abstract}
It is well known that amygdala plays a critical role in the development of stress responses and anxiety. It has been reported that nerve cells in the amygdala fire less by prolonged expiration, but clear evidence has not been shown. As a "ninja" technique, there is an extremely prolonged expiratory breathing technique called Okinaga, which keeps expiration for one minute. As a pilot study, I examined brain changes by Okinaga with fMRI. One ninja participated. He performed Okinaga for 30 minutes, after spontaneous breathing for 10 minutes. The last 5 minutes of each breathing session were compared by fMRI. A significant decrease in blood flow in the left amygdala and a significant increase in blood flow in the occipital and temporal lobes including the visual cortex and its surrounding area were observed. Although these results are interesting, suggesting that the prolonged expiratory breathing may lead to meditation, I need to investigate further by increasing the number of participants.
\end{abstract}

Keywords: Okinaga, fMRI; Breathing; Amygdala; Visual cortex

\section{Introduction}

Amygdala has been shown to play a critical role in the development of stress responses and anxiety [1]. As nerve cells in the amygdala fire less during quiet breathing with prolonged expiration [2], it is thought that the prolonged expiration will alleviate stress response and anxiety. Although the prolonged expiratory breathing is an important factor in yoga and mindfulness, the effect of prolonged expiratory breathing itself on the amygdala has not been investigated. In Japan, people known as "ninja" were most active 400 to 500 years ago and perhaps best known for their skill in conducting covert missions. Ninja has a breathing method called Okinaga which exerts extremely prolonged expiration. The duration of expiration reaches as much as 1 minute. Okinaga is originally intended to erase signs of oneself when stealing but is also thought to have been used to eliminate anxiety. Really, the old document knows that the ninja had overcome anxiety and stabilized the mind by Okinaga. The ninja often risked his life and appears to have mitigated the stress response in order to survive. "Oki" means "breathing", "naga" means "prolonged" in Japanese respectively. Ninja mastered this special breathing technique with training that began in early childhood. At the current time, there is only one genuine ninja who has repeatedly exercised training from childhood. In this study, I investigated the effects of Okinaga on brain function, using functional magnetic resonance imaging (fMRI).

\section{Methods}

\section{Participant and ethical considerations}

The procedures followed were in accordance with the ethical standards of the ethics committee of Mie University Graduate School of Medicine. One ninja participated in this study. He is 68 years old and healthy: He is only living ninja who trained from childhood. He gave his written informed consent to participate in the study.

\section{Breathing technique}

After spontaneous breathing for 10 minutes, the ninja performed Okinaga for 30 minutes. Okinaga is a very slow breathing, and expiration can require up to 1 minute.

\section{Laboratory environment}

The study was performed between 18:00 and 19:00 in an airconditioned MRI laboratory, maintained at $20-23^{\circ} \mathrm{C}$.

\section{fMRI data acquisition}

Data were recorded during the final 5 minutes of spontaneous breathing and final 5 minutes of prolonged expiration breathing. 
Functional images were acquired on a Ingenia 3.0T Omega HP (Philips Japan, Ltd.). A custom-built head holder was used to prevent head movement. The echo planar imaging sequence was acquired in 30 slices with slice thickness $=3.5 \mathrm{~mm}$, repetition time $=3000 \mathrm{~ms}$, echo time $=35 \mathrm{~ms}$, flip angle $=90^{\circ}$, field of view $=230$ $\mathrm{mm}$ x $230 \mathrm{~mm}$; sequence duration was $600 \mathrm{~s}$ for each subject; 200 time points were acquired; voxel dimensions were $2.4 \times 2.4 \times 3.5$ $\mathrm{mm}$.

\section{Statistical analysis}

A t-test was conducted via the attached software; the significance level was set to 0.05 .

\section{Result}

Figure 1 shows a section of the amygdala of the ninja. In the left amygdala, blood flow was significantly decreased during prolonged expiration breathing; blood flow decline was also observed in the left hippocampus. In contrast, blood flow increase was seen in part on the right amygdala, but no change was observed in most areas. Blood flow significantly increased in the temporal and occipital lobes including the visual cortex and its surrounding area (Figure 1).

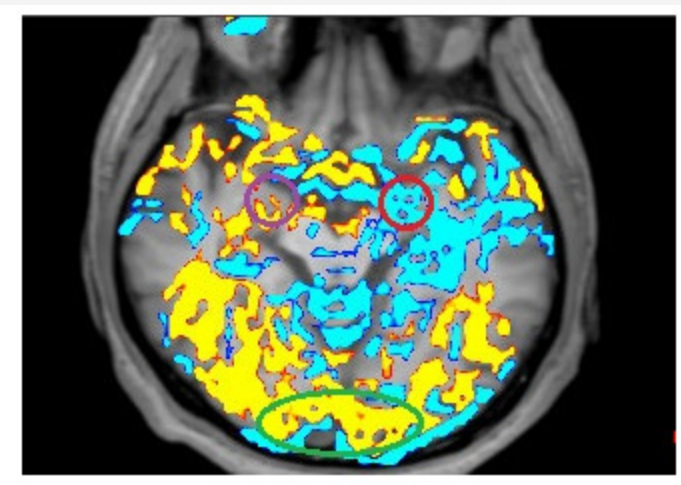

Figure 1: fMRI image of the ninja. red circle; left amygdala, purple circle; right amygdala, green circle; visual region.

\section{Discussion}

What I showed in this pilot study is the possibility that suppression of the left amygdala and activation of the occipital and temporal lobes may occur when the extremely prolonged expiratory breathing is acquired.

The amygdala causes emotion based on pleasure/discomfort sensory information, enabling an appropriate reaction [1]. Especially in cases of fear [3] and uncertainty [3], the amygdala activates the hypothalamus-pituitary-adrenal (HPA) axis [4] and the sympathetic nervous system [5], thus causing a stress reaction. Suppression of the amygdala function is important for alleviating the stress response. It has been reported that the amygdala is involved in various psychiatric disorders; in particular, it is important in depression and post-traumatic stress disorder [6]. In patients with depression, hyperactivation has been reported in the left amygdala, in response to emotional stimuli; this is interpreted as vulnerability to depression [7]. The metabolism of the left amygdala has been reported to correlate with blood cortisol [8] and corticotropin releasing hormone [9], indicating a close relationship between the left amygdala and the HPA axis.

In the 8-week Mindfulness Based Stress Reduction (MBSR) study, respective activity reductions were reported in the right amygdala [10] and left amygdala [11]. Meditation and yoga practitioners have been reported to exhibit a small right amygdala; these people are suggested to be more adaptable while experiencing stress [12]. Many studies have reported that mindfulness, yoga, and meditation cause decreased activity in the right amygdala; however, in some cases, a decrease in the activity of the left amygdala has also been observed. It is not clear what may cause these differences. Many reports show the model right amygdala mediates an initial, fast, perhaps automatic detection, followed by a more evaluative and discriminative response by the left amygdala [13].

In this study, it was notable that the ninja, in addition to exhibiting suppression of the left amygdala, showed enhanced activity of the occipital and temporal lobes. Among them, an increase in blood flow in the visual cortex is particularly notable. In fact, the ninja explained that his perception is sharp when performing Okinaga, as he performs Okinaga by envisioning various images. The sharpness of perception during Okinaga may be preparation for sudden attacks by enemies. Conversely, enhancement of visual cortex activity may indicate that Okinaga could be applied as meditation. The ninja explained that he performs Okinaga with a visual image such as the integration of the sun and the self. Ninja may have overcome anxiety by integrating with nature. Several previous fMRI studies have shown increased activity in visual regions during concentrative forms of meditation [14]. The 8-week MBSR training course enhances functional sensitivity within the auditory and visual networks, as well as promoting sensory processing; reflective surprises of sensory experiences are observed [14]. Mindfulness directs attention to breathing, thereby increasing awareness of it. Attention to breathing downregulates amygdala activation and increases amygdala-prefrontal integration [15]. Mindfulness may strengthen reasoning, controlled by the prefrontal cortex, and suppress sensations of overwhelming fear and anxiety in the amygdala. Whether this also applies during Okinaga is not yet clear. Thus, further verification is necessary.

\section{Conclusion}

Examination of the detailed mechanism is a component of future work; however, this study showed that, during Okinaga, suppression of the left amygdala and activation of the visual cortex occurred. This process may enable the ninja to control anxiety and suppress the stress response.

\section{Acknowledgement}

I thank Ryan Chastain-Gross, Ph.D., from Edanz Group (www. edanzediting.com/ac) for editing a draft of this manuscript.

\section{Conflict of Interest}

No conflict of interest. 


\section{References}

1. LeDoux J (2007) The amygdala. Curr Biol 17(20): R868-874.

2. Zhang JX, Harper RM, Frysinger RC (1986) Respiratory modulation of neuronal discharge in the central nucleus of the amygdala during sleep and waking states. Exp Neurol 91(1): 193-207.

3. Tobia MJ, Hayashi K, Ballard G, Gotlib IH, Waugh CE (2017) Dynamic functional connectivity and individual differences in emotions during social stress. Hum Brain Mapp 38(12): 6185-6206.

4. Pitkänen A, Pikkarainen M, Nurminen N, Ylinen A (2000) Reciprocal connections between the amygdala and the hippocampal formation, perirhinal cortex, and postrhinal cortex in rat. A review. Ann N Y Acad Sci 911: 369-391.

5. Macefield VG, James C, Henderson LA (2013) Identification of sites of sympathetic outflow at rest and during emotional arousal: concurrent recordings of sympathetic nerve activity and fMRI of the brain. Int J Psychopyysiol 89(3): 451-459.

6. Romeo RD (2017) The impact of stress on the structure of the adolescent brain: Implications for adolescent mental health. Brain Res 1654(Pt B): 185-191.

7. Zhong M, Wang X, Xiao J, Yi J, Zhu X, et al. (2011) Amygdala hyperactivation and prefrontal hypoactivation in subjects with cognitive vulnerability to depression. Biol Psychol 88(2-3): 233-242.

8. Drevets WC, Price JL, Bardgett ME, Reich T, Todd RD, et al. (2002) Glucose metabolism in the amygdala in depression: relationship to diagnostic subtype and plasma cortisol levels. Pharmacol Biochem Behav 71(3): 431-447.

9. Herman JP, Cullinan WE (1997) Neurocircuitry of stress: central control of the hypothalamo-pituitary-adrenocortical axis. Trends Neurosci 20(2): 78-84.

10. Hölzel BK, Hoge EA, Greve DN, Gard T, Creswell JD, et al. (2013) Neural mechanisms of symptom improvements in generalized anxiety disorder following mindfulness training. Neuroimage Clin 2: 448-458.

11. Farb N, Segal Z, Mayberg H, Bean J, McKeon D, et al. (2007) Attending to the present: Mindfulness meditation reveals distinct neural modes of self-reference. Soc Cogn Affect Neurosci 2(4): 313-322.

12. Gotink RA, Vernooij MW, Ikram MA, Niessen WJ, Krestin GP, et al. (2018) Meditation and yoga practice are associated with smaller right amygdala volume: the Rotterdam study. Brain Imaging Behav 12(6): 1631-1639.

13. Costafreda SG, Brammer MJ, David AS, Fu CH (2008) Predictors of amygdala activation during the processing of emotional stimuli: a metaanalysis of 385 PET and fMRI studies. Brain Res Rev 58(1): 57-70.

14. Berkovich-Ohana A, Harel M, Hahamy A, Arieli A, Malach R (2016) Alterations in task-induced activity and resting-state fluctuations in visual and DMN areas revealed in long-term meditators. Neuroimage 135: $125-134$.

15. Doll A, Hölzel BK, Mulej Bratec S, Boucard CC, Xie X, et al. (2016) Mindful attention to breath regulates emotions via increased amygdalaprefrontal cortex connectivity. Neuroimage 134: 305-313. 